\title{
Force-Free Models of Filament Channels
}

\author{
A. W. Longbottom \\ Mathematical Institute, University of St. Andrews, Fife KY16 9SS, U.K
}

\begin{abstract}
A fast multigrid method to calculate the linear force-free field for a prescribed photospheric flux distribution is outlined. This is used to examine an idealized model of a filament channel. The magnetic fields, for a number of different field strengths and positions, are calculated and the height up to which field lines connect along the channel is examined. This is shown to strongly depend on the value of the helicity of the system. A possible explanation, in terms of the global helicity of the system, is suggested for the dextral/sinistral hemispheric pattern observed in filament channels.
\end{abstract}

\section{Introduction}

Two of the prerequisites for the formation of solar prominences are that, first, an initial arcade structure must have already been formed above the polarity inversion line (PIL) and, second, that there must be a substantial component of the magnetic field along the PIL (Martin et al. 1994). Such a configuration is described as a filament channel. The overall magnetic field topology of these structures can be examined by the construction of force-free equilibria for given magnetic flux distributions at the photosphere. Such models, using the linear force-free approximation, are developed below allowing the global nature of filament channels to be explored.

\section{Linear Force-Free Fields and the Numerical Method}

In general a force-free equilibrium can be described by the equation

$$
(\nabla \times \mathbf{B}) \times \mathbf{B}=0
$$

where $\mathbf{B}$ is the magnetic field. This may be rewritten as

$$
\nabla \times \mathbf{B}=\alpha \mathbf{B}
$$

where $\alpha$ is a function of space and, in some sense, measures the helicity of the system. If $\alpha=0$ then the resulting equilibrium is current-free (or potential). If $\alpha=$ constant the equilibrium is linear force-free, and if $\alpha$ is a true function of space it is nonlinear force-free. The linear force-free field retains much of the detail of the nonlinear solution while being far easier to calculate. This approximation allows a substantially more realistic representation of the true equilibrium field than the current-free case. 
It must, however, be pointed out that the magnetic field may only be locally modelled by linear force-free equilibria, over regions of space where $\alpha$ is constant. Indeed, the linear solution is dependent on the size of the domain within which it is calculated. A number of theorems reguarding linear force-free fields are well established (Berger 1985): 1. For a given size of domain and boundary conditions a series of resonance points occur, as $\alpha$ is increased/decreased from zero, where the helicity and magnetic energy go to infinity. 2. For a given helicity $(\alpha)$ the linear force-free field is the lowest energy equilibrium compatible with the fixed boundary conditions. 3. All solutions with $\alpha$ less than the value of the first resonance point are linearly stable to ideal MHD modes.

Here the linear force-free equation is solved numerically by the method of Finn et al. (1994) rewriting (1) as

$$
\nabla^{2} \mathbf{A}=\alpha \nabla \times \mathbf{A},
$$

where

$$
\mathbf{B}=\nabla \times \mathbf{A} .
$$

The numerical box is a cube with normalized dimensions $-1<x, y<1,0<z<$ 2 and has a resolution of up to $129^{3}$ grid points. For a given vertical component of magnetic field prescribed on the base of the box (the photosphere) a Poisson equation is solved, on the base, to give the values of $\mathbf{A}$ on that face. The elliptic equation (2) is then solved directly within the three-dimensional domain. The boundary conditions on the faces, other than the photospheric base, are that the normal component of magnetic field vanishes and $\nabla \cdot \mathbf{A}=0$. The second of these guarantees that when (2) is solved with the given boundary conditions $\nabla \cdot \mathbf{A}=0$ everywhere within the computational domain.

If classical iterative methods (such as SOR) are used to solve the equations, convergence towards the solution quickly saturates once short wavelength errors are smoothed. In fact, the number of iterations to gain a given accuracy scales like the square of the number of grid points. Thus, doubling the resolution requires 64 times the number of iterations.

Here linear multigrid methods (Longbottom et al. 1998) are used to speed up the rate of convergence of the classical scheme. Multigrid methods smooth all wavelength errors simultaneously so convergence does not saturate. The number of iterations to gain a given accuracy scales independently of the grid size.

\section{Results}

In trying to model a filament channel an idealized flux distribution is used. This consists of two elongated regions of equal and opposite flux emerging from the photosphere, forming the overlying arcade, together with two concentrated regions of flux at either end of the arcade, representing an underlying component of the field directed along the length filament channel. This geometry together with a typical calculated field is shown in Figure 1. Ratios of concentrated to elongated flux per unit area between 2 and 24 have been taken, with ratios $a / b$ of separation of concentrated to elongated flux regions of 1 or 2 . 

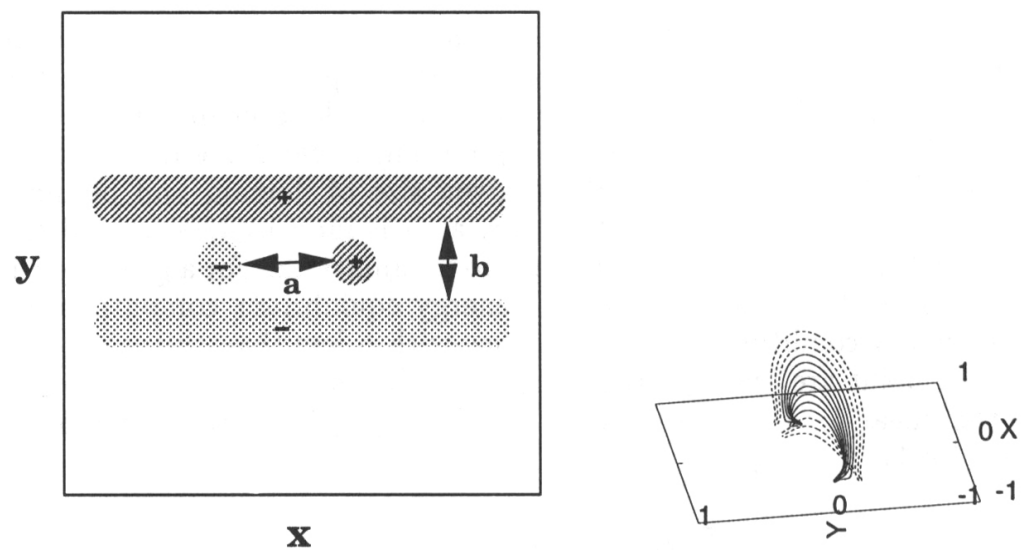

Figure 1. (a) The flux distribution on the photospheric base. (b) Field lines for the case $a / b=2$, flux ratio $=12, \alpha=-2$.

For each case the linear force-free field between the the first (positive and negative) values of the resonant $\alpha$ have been calculated. An examination of the field line topology then gives distinct regions of field line connectivity. The separatrix surface enclosing all field lines that connect the two concentrated sources can be calculated. The maximum separatrix height (always along the line rising vertically from the centre of the base) as a function of $\alpha$, is plotted in Figure 2.
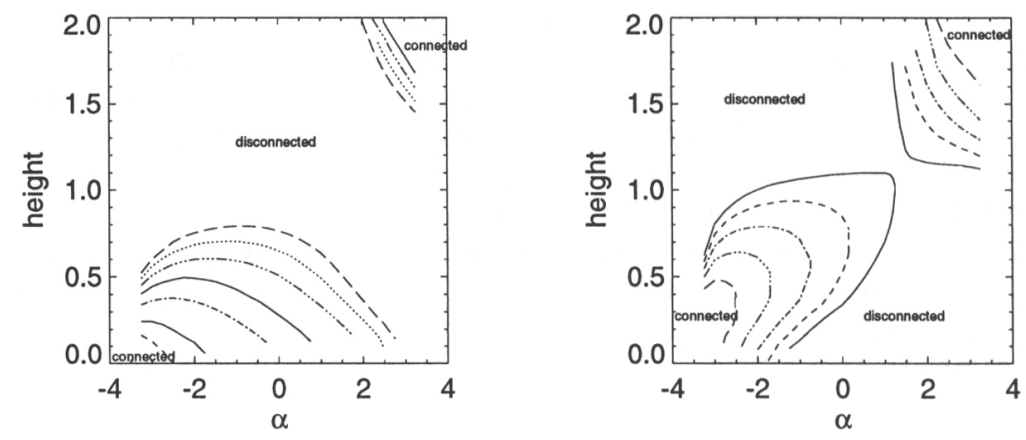

Figure 2. The height of the separatrix surface for (a) $a / b=1$ and (b) $a / b=2$.

The results for $a / b=1$ are shown in Figure 2a. This represents the case where the concentrated flux sources are separated by the width of the filament channel. With increasing height, the curves represent flux ratios of $2,4,8,12,16$, 20 and 24 . The curves for large $\alpha$ and height are due to the boundary conditions on the top and sides of the computational domain and do not represent physically relevant solutions. For this flux distribution a value of helicity $(\alpha)$ may always 
be found for which field lines will connect the two concentrated flux sources, forming a component of field along the channel.

For the potential case $(\alpha=0)$ considered by MacKay and Priest (1998) this would not be the case. Only when the concentrated flux becomes large with respect to the arcade flux do field lines connect the two concentrated sources.

Figure $2 \mathrm{~b}$ shows the equivalent results for the case $a / b=2$ (the concentrated flux sources are separated towards the ends of the filament channel). The curves from left to right represent flux ratios of 8,12,16, 20 and 24. Again the curves for large $\alpha$ and height are due to the top and side boundary conditions. In most cases for a given $\alpha$, there is now both a lower and upper bound to the separatrix surface giving a closed volume above the photospheric boundary within which field lines connect the concentrated sources along the filament channel. It can also be noted that, as the concentrated sources are now further separated, greater strengths are required to connect them.

A plot of the field lines for the case $a / b=2$, flux ratio $=12, \alpha=-2$ is shown in Figure 1b. Field lines connecting the two concentrated sources are shown solid and those connecting the arcade dashed. It is interesting to note that this represents a dextral filament channel which is preferentially observed in the northern hemisphere. Figure $2 b$ shows that this topology (Figure 1a) has a bias towards negative values of $\alpha$. Similarly sinistral filament channels (observed preferentially in the southern hemisphere) show a bias towards positive values of $\alpha$. This agrees with the corresponding hemispheric variation of $\alpha$ observed by Pevtsov et al. (1995). For further details see MacKay and Longbottom (1998).

\section{Conclusions}

This paper outlines a method for the fast generation of linear force-free equilibria for a given magnetic flux distribution at the photospheric boundary. This has been used to model the global topology of a filament channel. It is shown that both the strong component of field along the channel and dextral/sinistral hemispheric pattern can be reproduced by the appropriate choice of helicity, this choice agreeing with the observed hemispheric pattern of helicity.

\section{References}

Berger, M. 1985, ApJS, 59, 433

Finn, J., Guzdar, P., and Usikov, D. 1994, ApJ, 427, 475

Longbottom, A., Fiedler, R. and Rickard, G. 1998, A\&AS, submitted

MacKay, D. and Priest, E. 1997, Solar Phys., 167, 281

MacKay, D. and Longbottom, A. 1998, ApJ, in preparation

Martin, S., Bilimoria, R and Tracadas, P. 1994, in Solar Surface Magnetism, R

Rutten and C. Schrijver, Springer-Verlag, New York, 303

Pevtsov, A., Canfield, R. and Metcalf, T. 1995, ApJ, 440, L109 